\title{
Healthcare workers' experiences of HIV testing in Tshwane, South Africa
}

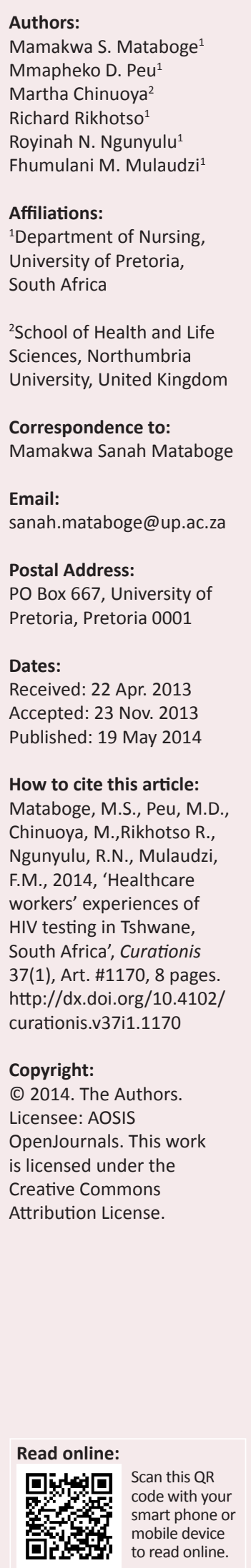

Background: In an era when antiretroviral (ARV) therapy has become part of the Human Immunodeficiency Virus (HIV) prevention strategy, early testing and introduction to ARVs is critical for improving public health outcomes in general and, in particular, the lives of people living with HIV. South Africa has the highest number of people living with HIV as compared with the rest of the world. Initiated voluntary HIV counselling and testing and providerinitiated counselling and testing (PICT) are required in order to increase the uptake of HIV testing.

Objectives: To explore and describe the experiences of healthcare workers who are themselves in need of HIV testing.

Method: A descriptive, exploratory design was used. In-depth interviews were conducted with the 26 healthcare workers who were involved in HIV testing in the Tshwane district of South Africa. The participants were sampled purposively from two healthcare settings. A thematic framework was used for data analysis.

Results: There was a complication with regard to PICT as healthcare workers felt they could not initiate HIV testing for themselves and or their work colleagues without their confidentiality being compromised. This was complicated further by both the perceived and actual fear of stigmatisation and discrimination. It was difficult for qualified staff to support and encourage the uptake of HIV testing by students nurses as this was seen, albeit incorrectly, as targeting the students in a negative manner.

Conclusion: There is a need for accessible HIV testing policies for healthcare workers in order to increase access to HIV testing and prevent the progression of the disease.

\section{Introduction \\ Background}

South Africa has the highest number of people living with the Human Immunodeficiency Virus (HIV) and Acquired Immune Deficiency Syndrome (AIDS), with 5.63 million people infected, the majority of whom are women (Department of Health [DoH] 2012:22). Many South Africans do not know their HIV status, despite the routine promotion of voluntary HIV counselling and testing (HCT). Clients diagnosed only during antenatal care are a testament to the fact that most South Africans do not access HCT timeously (DoH 2012:22). This indicates that there are many missed opportunities, as a large number of clients leave the clinic without HCT. To optimise HIV counselling and testing and to maximise the numbers accessing HCT in clinical settings, provider-initiated counselling and testing (PICT) was introduced in order to reach out to clients who do not opt for self-initiated counselling and testing. PICT and HCT are voluntary and clients give informed consent for HIV testing. In South Africa, PICT services are provided by trained nurses, doctors and other allied healthcare workers. HIV testing is part of the primary healthcare services that promote the health of individuals and prevent the spread of the disease amongst various communities, as well as helping with the prevention of mother-to-child transmission (PMTCT). In South Africa, PICT is meant to be a gateway for all clients to access comprehensive healthcare (DoH 2012:40). The provision of PICT has become part of the HIV testing routine that is provided in over 4500 health facilities, including mobile units (DoH 2010:11); and healthcare workers are at the core of implementing HCT policies and initiatives. HCT and PICT remain the cornerstone for the prevention of HIV and AIDS in South Africa (Kalichman \& Simbayi 2003:442) and for achieving the aspiration of an HIV-free generation. In the Tshwane district, HIV healthcare workers are part of a community of practice $(\mathrm{CoP})$. A CoP is a group of people who share a common purpose and who learn how to be effective and who improve performance by meeting regularly to discuss common concerns in order to deepen their knowledge (Wenger, McDermott \& Snyder 2002:4). In the healthcare arena, a CoP is an informal means through which knowledge is shared in order to improve patient care (Ranmuthugala et al. 2011:4). Healthcare 
workers are also professionals and are guided by ethical and professional codes of conduct.

Human rights and access to justice for pregnant women during PICT is one of the priority areas in the National Strategic Plan on HIV, STIs (sexually transmitted infections) and TB: 2012-2016 policy (DoH 2012:12). In an era where antiretroviral (ARV) treatments are part of the HIV prevention strategy, HCT assists in the early detection and diagnosis of the virus and, if the client is HIV positive, clinical decisions will be made for initiation of ARVs (Wheelock et al. 2012:2). HCT is an entry point to a comprehensive range of care. This care involves prevention, treatment, care and support (DoH 2010:3). According to Leon et al. (2010:220), PICT for HIV was suggested as a possible strategy to expand HIV testing and could be offered routinely during voluntary counselling and testing (VCT) campaigns, mobile VCT services, home-based HIV testing initiatives and self-testing initiatives. By using client-initiated counselling and testing, with the upscaling of PICT, the South African government was aiming to test 15 million people by June 2011 and to encourage the same people to have repeat tests at least once a year (South African National AIDS Council [SANAC] 2010:9). This is in line with the National Strategic Plan of South Africa. The National Strategic Plan on HIV, STIs and TB's aim is 'to reduce new HIV infections by at least 50\% using a combination of prevention approaches' which include 'initiating at least $80 \%$ of eligible patients on $\mathrm{ART}^{\prime}$ and 'reducing self-reported stigma and discrimination related to HIV and TB by $50 \%$ ' (DoH 2012:12). This policy initiative aimed at creating jobs for 8000 volunteers, who were to be trained to provide HCT in return for a stipend. However, the positioning of lay counsellors in the South African healthcare system within the multidisciplinary team is to date - 2013 - not formal and translates into counsellors being unsure of their position within the health team. In addition, some do not receive support from any nurse managers (Rohleder \& Swartz 2005:403). International and national funding to facilitate the promotion of counselling and testing-promoted vertical programmes, namely, programmes focusing on HIV and AIDS only in primary healthcare settings, has been successful in scaling up HIV testing (Duber et al. 2010:13). There are similarities between PICT and HCT but also differences, as are represented in Table 1 (Makhunga-Ramfolo et al. 2011:9).

Both PICT and VCT, as presented in Table 1, require confidentiality and consent during testing. Healthcare workers are expected to ensure the provision of these factors to clients and evidence shows that they do so. The success of the implementation of PICT for pregnant women is clear in the significant increase in the number of women tested for HIV during pregnancy (Gruskin, Ahmed \& Ferguson 2008:26). In South Africa, HCT and PICT have significantly increased the number of TB patients who receive counselling and testing (Pope et al. 2008:195). The positive outcome of PICT are the reduced number of HIV-related deaths in sub-Saharan Africa: a decrease of $18 \%$ between 2004 and 2009 from 740000 to 610000 (Topp et al. 2012:1). A study conducted in South Africa confirmed that PICT is also a path toward diagnosing HIV-positive children, as $90.2 \%$ of the children who have been diagnosed have been diagnosed through the implementation of the strategy (Horwood et al. 2009:9). However, the accessibility of HCT is not uniform throughout the world. In more developed countries, about $20 \%-30 \%$ of individuals living with HIV are unaware that they are HIV positive because they only come for HCT once their symptoms are clinically very advanced (Obermeyer \& Osborn 2007:1762). Despite the availability of ethical guidelines related to PICT provision, there are indications that some healthcare providers still conduct routine HCT without informing the patients and without their consent (Obermeyer \& Osborn 2007:1768).

The implementation of HCT, as provided by trained volunteers and healthcare workers, presents many challenges, including staff shortages (Soai 2012:1). Nurses who provide PICT experience challenges that include, amongst others, the distance travelled by clients, fears of occupational risks to HIV, clients waiting in long queues as a result of limited resources, as well as a lack of professional integrity (Soai 2012:1). Healthcare workers are at risk of HIV infection from occupational exposure to blood-borne pathogens during their daily work activities (Zungu \& Sanni 2011:488) because of poor compliance with the universal precautions whilst busy with their professional duties (Akinboro, Adejumo \& Olowekere 2012:3). Healthcare workers also experience challenges related to the counselling process. HIV counselling takes a longer time than ordinary counselling - more than what is estimated by some of the professional nurses - and this extra time affects the number of patients seen per day (Rohleder \& Swartz 2005:403). When having to deal with HIV counselling, healthcare workers find they have to spend time attending to a client's emotional problems first before actually providing counselling. The approach of some professional healthcare workers to general

TABLE 1: The similarities and differences of patient-initiated and voluntary counselling and testing.

\begin{tabular}{ll}
\hline PICT & VCT \\
\hline $\begin{array}{l}\text { Individual is seeking medical care and HCT is recommended and performed by } \\
\text { healthcare worker during consultation }\end{array}$ & Individual chooses to seek HCT \\
$\begin{array}{l}\text { Services provided are confidential and documented in medical record to ensure } \\
\text { continuity of care }\end{array}$ & Anonymous or confidential services may be offered \\
$\begin{array}{l}\text { Primary focus is on identifying HIV-infected people and linking them with prevention, } \\
\text { care and treatment services }\end{array}$ & $\begin{array}{l}\text { Primary focus is on preventing HIV acquisition through risk assessment, risk reduction } \\
\text { and testing }\end{array}$ \\
$\begin{array}{l}\text { Verbal consent is required and should be documented in the patient record } \\
\begin{array}{l}\text { First user of the test result is the healthcare worker to make a correct diagnosis and } \\
\text { provide appropriate treatment }\end{array}\end{array}$ & $\begin{array}{l}\text { Written consent or thumb print for illiterate clients is required } \\
\text { First user of the test result is the client, who uses the information to make personal life }\end{array}$ \\
\hline
\end{tabular}
provide appropriate treatment 
counselling and testing disregards the patient's emotional well-being in order to save on consultation time (Rohleder \& Swartz 2005:402). Opportunity to counsel, as well as the time needed to counsel, are challenges that need careful management or they could have a detrimental effect on HIV and AIDS counselling as a standard of care (Soai 2012:2). Sometimes, healthcare workers experience the dilemma of having to discuss the results of HCT as well at the first counselling session. The reason for this is that clients often do not return for the results of the HIV test and the posttest counselling, therefore the first counselling session is the only opportunity to counsel the client on a positive HIV test (World Health Organization [WHO] 2007:12). The WHO also reported the challenges of HIV disclosure and the stigma of being identified as HIV positive as being a major reason for the slow uptake of PICT and lack of follow-up consultations.

\section{Problem statement}

Healthcare workers in sub-Saharan Africa reportedly perceive themselves as being exposed to a higher risk of HIV infection because of the high rate of HIV infection in the larger population (Zungu \& Sanni 2011:488). A number of studies have been conducted about HIV testing attitudes, experiences and the stigma of clients, but little is known about the personal experiences of healthcare workers with HIV testing in the Tshwane district of South Africa (Smit 2005:23). These are the very people who are responsible for the provision of the HCT services.

\section{Aim of the study}

To explore and describe the experiences of healthcare workers of HIV testing in Tshwane, South Africa.

\section{Trends}

A study conducted amongst health workers on HIV status in two healthcare institutions in Gauteng indicated that prevalence was highest amongst the student nurses (Connelly et al. 2007:117) and those aged $25-34$ years $(15.9 \% ; n=1493)$ and highest.

\section{Research design}

A qualitative research approach was used in this study in order to explore the experiences of healthcare workers with regard to HIV testing in Tshwane, South Africa. The participants were members of the HIV CoP who were selected purposively because they were responsible for the implementation of HIV testing policies, and they worked in HIV testing centres.

The research protocol was reviewed by a University Ethics Committee who granted ethical approval (reference number 75/2012). The request with regard to participation in the research, briefing on the research aim and objectives and agreement regarding participation and the venue for the interview were done telephonically. On the day data collection was done, the participants were given the information sheet relating to the study and study process and had the opportunity to ask questions about the study. They then agreed to participate voluntarily and indicated this by signing the informed consent form before participation. An interview guide was used to explore the participants' experiences of HIV testing. The interviews were unstructured to allow for an in-depth exploration of the key themes of the study, in particular the experience of utilising PICT and HCT amongst healthcare workers in HIV clinic settings. All interviews lasted about 40 minutes and were recorded and transcribed verbatim. The transcripts were then subjected to a thematic framework as outlined by Ritchie and Spencer (1993:173-194). The thematic framework follows a six-step process of analysing interview transcripts: familiarisation, identifying a thematic framework, indexing, charting, mapping and interpreting. Themes that emerged from the data addressed how participants negotiated the boundaries between HCT and PICT.

\section{The sample}

There were 26 participants in the study. These included clinic nurses $(n=3)$, lecturers $(n=7)$, nursing students from the two universities $(n=3)$, support workers $(n=3)$, lay counsellors $(n=7)$ and allied healthcare workers $(n=3)$. Most of the participants were women $(n=22)$, with only a few being men $(n=4)$. This is not surprising as the caring profession in South Africa and elsewhere is dominated by women. Participants were engaged to discuss the key themes, namely, (1) the boundaries between HCT and PICT for student nurses, (2) the boundaries for HCT and PICT for qualified nurses and (3) structural issues.

\section{Results \\ Boundaries for student nurses}

Client-initiated HIV testing is initiated by the client who comes to the clinic for HIV tests or other services. However, the identity of who was a client became blurred when healthcare workers themselves were the clients: an example being nursing students or qualified nurses initiating HIV testing. They then became potential clients. Some healthcare workers viewed HCT as being vitally important for professionals and these healthcare workers felt they should initiate testing for other healthcare workers who would then become clients. Professionals were also seen as being responsible for their own sex life. Young student nurses, in particular, were perceived as being at risk as a result of practising risky sexual behaviour. This is evident in the following quote:

'I think they need to be targeted; remember they are away from home where they were under the care of their parents and are now living on their own. They've got their own freedom and it is up to an individual person. At times they do not know their status and they meet new partners, so I think they need to be targeted'. (P5, Female, 43)

Although HIV testing was seen as being really important for student nurses, it was difficult for qualified healthcare workers to encourage PICT for students as this was seen 
as discriminatory and 'targeting'. Most qualified healthcare workers viewed HCT as being important for all people, including qualified staff and student nurses. One healthcare provider indicated that:

'No, like I say, when they conduct the campaign they organise it for everybody. It is a voluntary effort'. (P10, Male, 33)

Some healthcare providers viewed the issue of confidentiality within the clinic setting and amongst themselves as a problem and this restricted them from providing full support to nursing students and qualified staff who tested HIV positive. Although they have in-house counselling services, they preferred to refer HIV-positive students to other HIV counselling centres, away from their placements, as is indicated below:

'The support is available in the counselling centres and if I know that they are positive I can assist where I can, but with HIV we are restricted by confidentiality issues'. (P15, Female, 35)

Suggestions from student participants about who to approach for support should they test positive ranged from their lecturers, with whom they had a close relationship (e.g. like a mentor), to other people they felt comfortable with. One student said:

'I think I would go to the lecturer whom I know I am close to; because in 1st year we are each allocated a lecturer who serves as a mentor, I feel if we build a good rapport at first level, then would come [sic] and talk with them. Not to say they cannot talk to me at 2nd to 4th level'. (P5, Female, 43)

As noted above, lecturers were perceived as being qualified to support students, particularly if they had mentored a student for a long time and had formed a close relationship with the student. Despite the students' wishes to discuss potential HIV testing and counselling with their mentors, a qualified healthcare worker said:

'We have the student counselling department where we refer them to when they have problems'. (P5, Female, 43)

There seemed to be some disagreement about who students should talk to about potential HIV diagnosis within HIV testing centres. Some qualified staff, as noted above, suggested that they would prefer to refer students to student counselling departments. Qualified healthcare workers were unclear as to whether they thought nurse-lecturers had adequate skills to deal with students if the students presented with problems related to HCT. When asked if they discussed HCT with students, one healthcare worker said:

'I cannot really say that discussing HIV testing with students is not happening because from my point of view from the college, when a student is ailing we always make time and do follow up on them. We always follow up and not just jump to conclusion. It first starts when a student starts being absent in class frequently, that's where we pick up that there is a problem and we follow up and start asking what could be the problem'. (P10, Male, 33)

Qualified staff reported instances where students often missed classes and became increasingly unwell, with suspected HIV infections. The discussion that follows focuses on the challenges experienced by qualified staff in initiating HIV testing for themselves or their peers.

\section{Boundaries for qualified nurses}

The level of risk for HIV infection was considered low amongst healthcare professionals. Although they were skilled in initiating testing for their clients, there was less enthusiasm with regard to initiating testing for themselves. Even though they are involved with PICT, qualified healthcare workers simply did not perceive themselves as being at risk of HIV infection, as was communicated by one participant:

'What is even worse with our practitioners is that they do not see themselves in the eyes of HIV infection. It's like it is not for them but for patients around them'. (P15, Female, 35)

The participants spoke about occupational HIV risks. Although HCT provides clear guidelines regarding postexposure prophylaxis, there was an indication of a lack of knowledge amongst the participants regarding the occupational risks, such as those arising from needle-stick injuries. The breakdown of understanding was in terms of finding out if a needle stick had infected the healthcare worker; it was as if the healthcare worker was automatically considered to be infected and incapable of working. The discrimination of work colleagues was immediate, as was experienced by one participant who had experienced a needle stick:

'Oh I've pricked myself with the needle and the patient said: "Sister, I am HIV positive". They [healthcare colleagues] thought if I pricked myself with a needle, it was obvious that I was HIV

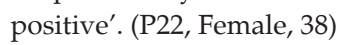

The above participant then detailed the discrimination she experienced from her colleagues:

'We were taught about it [needle stick]. But they just assumed that when I'm needle pricked [sic] with an HIV-positive needle I am also HIV positive. There and there, they did not even want me to write in the maternity register. They offered to write on my behalf in the register... My colleagues started to discriminate against me'. (P22, Female, 38)

Post-test counselling should be given in an ethicallyacceptable manner. However, some healthcare workers who had been exposed to needle-stick injuries from HIV-positive patients reported that they were given the results away from their place of work. One healthcare worker reported that she was informed of her results telephonically when not at work:

'I received pre- and post-test counselling and they told me the results when I was off the hospital premises, which was very uncomfortable'. (P22, Female, 38)

Unlike a routine HCT and PICT situation, where HIV results are communicated within the context of post-test counselling, this participant reported that her results were communicated via the telephone and that it included no post-counselling procedures.

Healthcare workers who accessed counselling and testing and had a positive HIV diagnosis reported that they would 
not disclose their status to their employer or colleagues for fear of perceived stigma and discrimination. Although their employers had employee-assistance programmes that accommodated their HIV status, for example, by placing them in low-risk units, the participants reported that they felt they could not disclose their status for fear of stigmatisation. When they did disclose their status, they were assisted:

'Our staff could come and talk to us about their HIV status but they chose to keep quite. We would allocate them in the wards that are less infectious, put them on prophylactic treatment to strengthen their quality of life. The few that came to us and disclosed [their status], are allocated appropriately and their health has improved'. (P7, Female, 58)

Lack of resources, stigma and confidentiality were the barriers to healthcare workers accessing HIV testing within their workplace. When healthcare workers knew they were positive, they would not disclose their status to colleagues in the workplace, or even to the supervisor. The perception was that healthcare workers involved in HCT and PICT did not have adequate support from their employers as they were concerned about being stigmatised. Concerns and fears of being stigmatised by work colleagues were mentioned repeatedly. This impression was based on the attitudes of some staff members toward them clients. Healthcare workers did not themselves want to endure this stigma. The fear of being stigmatised leads to their not accessing HCT and, therefore, colleagues do not initiate PICT with their peers. The views on stigmatisation were emphasised:

'Yes, I believe healthcare workers do still stigmatise people living with HIV and AIDS, even in my unit'. (P15, Female, 35)

\section{Structural issues}

Structural issues were discussed within the context of the training policies for healthcare workers, as were the accessibility and availability of resources to healthcare workers in order to access HIV testing and counselling. One important factor identified by staff was the lack of dedicated resources to support staff in order to initiate HCT and to assist colleagues to access HIV testing as part of the PICT services. One participant said:

'I feel we need to do introspection [sic] because we do not have those services [HIV testing services] for staff'. (P23, Female, 40)

The statement above highlights that there were limited HIV testing services that addressed the needs of healthcare workers; and that the healthcare workers perceived themselves as being neither clients nor service users of their work-based HIV testing services. Compounded by fears of being stigmatised and the perceived lack of confidentiality, most participants reported that qualified healthcare workers did not utilise their work institution to access treatment for HIV, but rather relied on their private doctors until their funds were exhausted. These feelings were expressed starkly as follows:

'Our staff do not agree to come and take treatment at our hospital. They use their medical aid until it's exhausted and there is nowhere to get medication from ... they default and die' (P19, Female, 36).
The fear of being stigmatised resulted in limited adherence or access to ARV treatment, as has been noted above. This was despite the fact that some of the qualified healthcare workers were employed in areas where ARVs were freely available to clients.

\section{Discussion}

The participants of the current study, who are healthcare workers, occupy problematic positions and identities as they are the providers and implementers of HIV testing, yet they are also potential clients of the HCT services. Their identity as providers and/or implementers of the HIV counselling service is complicated by perceived fears of stigmatisation and breach of confidentiality by colleagues should they test HIV positive. These fears limit them from initiating HCT for themselves as potential clients of the service or PICT for fellow healthcare providers in order to avert stigma and discrimination if they test positive (Musheke et al. 2013:6). As promoters of health, they seemed unable to implement HIV testing initiatives in their own lives, resulting in missed opportunities for diagnosis. In a study by Connelly et al. (2007:115), there was an $11.5 \%$ prevalence of HIV and AIDS, with $19 \%$ having an average CD4 count of 200 cells / $\mu \mathrm{l}$, amongst health workers at two healthcare facilities in Gauteng, South Africa. Having healthcare providers who are not healthy becomes a problem, as was reported by Davhana-Maselesele and Igumbor (2008:72), where healthcare providers in the Limpopo Province presented with depression whilst providing care to people living with HIV.

Similar findings emerged in this study. It was evident that although healthcare workers provided HCT and PICT, some of them experience challenges with the initiation of HCT for themselves as healthcare workers. These healthcare workers were members of the HIV CoP in the Tshwane district in the Gauteng Province of South Africa. They were responsible for the implementation of HCT and PICT in order to reduce the impact of the HIV epidemic in line with government policies and frameworks, but they never tested themselves.

All healthcare workers should undergo VCT as standard policy (Smit 2005:24), but this is far from being attained. To start with, there are no dedicated sites for the counselling and testing of healthcare workers. What emerges is that wellness centres are needed where healthcare workers can report for any work-related injuries, including exposure to HIV infection risks. There is a need to set goals and policy to enable healthcare workers to access HIV testing, as was suggested by Mavedzenge et al. (2011:25-29). These goals and policies would clarify where alternative non-mandatory, non-coercive sites exist in order for the healthcare worker to access occupational testing programmes and to ensure confidentiality. Other methods of providing HIV testing to healthcare workers could include managers assisting healthcare workers in attending HCT sites outside of their work areas, such as with private providers of their own choice; service provider exchange where an unknown 
provider does HCT within the workplace; mobile testing; or home-based testing. The latter HCT provision could, however, lead to stigmatisation when community members become aware of a mobile unit which targets only healthcare workers' residential addresses (Mavedzenge et al. 2011:25-29).

Healthcare workers also experience stigmatisation amongst themselves. Experiences and perceptions of being stigmatised may discourage people from seeking medical help at an early stage. The participants mentioned repeatedly that stigmatisation affected healthcare workers' integrity and the confidentiality of clients. To support this notion, Kardas-Nelson (2012:1) states that stigma and discrimination have long prevented people from accessing HIV- and AIDS-related services, an example of this being nurses who think negatively of people living with HIV having a great influence on whether or not their colleagues access such services. This paper shows that stigmatisation also affects healthcare workers. Lack of resources is a further obstacle and a concern in the management of HIV and AIDS. A study by Peu, van Wyk and Botha (2008:16) showed that poor access to healthcare services in South Africa makes it difficult to promote the health of the community. They also found that the shortage of resources and lack of treatment affect the management of HIV and AIDS. In response to the shortage of resources, various measures were implemented. These measures included various counselling and testing procedures and the training of healthcare workers - all aimed at reducing the impact of HIV and AIDS in South Africa. However, despite these efforts, there are limited opportunities for healthcare workers to initiate and access HCT or offer PICT within their workplaces.

Healthcare workers, especially those who are involved in caring for HIV and AIDS patients, are exposed to multiple ways of contracting HIV and this knowledge affects their performance as service providers. These healthcare workers are qualified staff who were trained and qualified to practise within the legal framework of their occupation. The HCT provision sites expanded from 7700 in 2007 to 22400 in 2010 which immediately exposed healthcare workers to a greater percentage risk of contracting HIV during the provision of HCT services. These sites successfully facilitated the enrolment of 6650000 people into antiretroviral therapy programmes, as was intended with the availability of PICT and HCT (WHO 2011:5). Furthermore, through their efforts and by working within the scope of their profession, healthcare workers have facilitated the success of PMTCT programmes with the diagnosis of HIV amongst the general public and a successful roll-out of ARVs to the public in South Africa. In sub-Saharan Africa, significance progress has been made in providing access to ARV therapy (Topp et al. 2012:1). The cost and challenge has been the lack of HCT or PICT for healthcare workers themselves, which was made even more difficult for them when there was a lack of employer support. Though healthcare workers (nurses) were the ones in the frontline to ensure access to PICT and HCT for clients, they were not being rewarded. The focus on HCT and PICT uptake by healthcare workers was lacking, even though the risk of contracting HIV in the workplace was higher.

Testing as a preventative measure is undoubtedly not enough: assessing a client's risk, how they feel and making them aware of their need to change their behaviour are equally important. A service that provides information that creates relationships with individuals and also works with clients must keep finding appropriate ways of delivering the service to create a stable healthy population (Hobbs 2011:1). There are global political shifts in HIV prevention strategies, with highly-effective antiretroviral therapies (HAART) increasingly becoming part of the HIV prevention strategy. South Africa's aim is that $80 \%$ of HIV-infected people should have access to ARVs between 2012 and 2016 (DoH 2012:12). It is through the combined efforts of healthcare workers and the public that aspirations of an AIDS-free generation can be envisaged, with zero new HIV infections as a result of increased PICT uptake. However, there is a need for funding. The financial impact of HIV and AIDS on the South African economy was confirmed by the inclusion of South Africa as one of the 12 sub-Saharan countries selected to receive assistance from the US President's Emergency Plan for AIDS Relief (PEPFAR) (Duber et al. 2010:4).

The Department of Public Service and Administration (DPSA), in partnership with the DoH and the South African National AIDS Council (SANAC), embarked on a national HCT campaign to counsel and test 1.2 million public servants: aimed at reducing the statistics of untested and untreated cases of HIV and AIDS. As its focal point, the campaign stated that long life was a guarantee for those who tested early and were treated early; it also would help identify and treat tuberculosis (TB), which attacks people with immune systems weakened by undiagnosed and untreated HIV infection (DoH 2012:12-14). Partnership with employers is needed in the health sector. The sector could ill afford the destabilisation of its workforce because of undiagnosed and untreated HIV infection amongst its own workforce. HIV and related diagnoses have destabilised the health system, the social protection system, education, industry, the formal and informal agricultural sectors, civil society and even the political system. The involvement of employer organisations has contributed to the national effort to reduce the impact of HIV and AIDS by mobilising their member enterprises, managers and workers to join the war against HIV and AIDS (Conference Report, Arusha 2012:1-2). Healthcare workers, particularly nurses, working directly with HIV clients in high-risk units, are at risk of contracting HIV and need to be prioritised in order to ensure that they can access counselling and testing. The workplaces should provide support and encourage HIV testing annually for all staff members, students and clients. This would encourage them to take advantage of regular testing. Healthcare workers need to know they are protected in terms of confidentiality and can continue work, regardless of their status. Li et al. (2007, as quoted in Davhana-Maselesele \& Igumbor 2008:72) made it clear that nurses need to remain healthy as the care of HIVinfected patients is dependent on their wellbeing. 
Training remains an important component of empowering healthcare workers about the risks of contracting HIV and the need for the uptake of HCT in order to remain healthy. Just like the general populace, healthcare workers face the risk of HIV infection in both their personal and professional lives (Zungu \& Sanni 2011:488). Approximately 3 million healthcare workers of the 35 million worldwide experience percutaneous exposure to blood-borne viruses, including HIV (Akinboro et al. 2012:2). All healthcare workers should treat any blood and other body fluids from all patients as potentially infectious. In the case of contact with patients' secretions, testing for HIV infection becomes the first step for post-exposure prophylaxis. However, it remains controversial for a variety of reasons, such as the fear of being stigmatised and the resulting discrimination. In Uganda, more than half of the healthcare workers reported that they have experienced at least one needle-stick injury per year (Nsubunga \& Jaakkola 2005:773). Findings from Kenya also show that HIV infections and AIDS are having a negative effect on the nursing workforce because of the absence of adequate workplace programmes for healthcare workers. Meaningful actions need to be provided, including counselling and the provision of ARV treatment for healthcare workers. The magnitude of the impact of HIV on the nursing workforce is still unfolding. The stigma associated with the disease makes it difficult for accurate data to be obtained about its effect on nurses because of the failure to report an HIV-positive status (Munjanja, Kibuka \& Dovlo 2005:7-8). They also lacked clear guidelines regarding how to initiate PICT for their peers, as is the case in this study. A diversified service that caters for everyone will encourage healthcare workers to undergo HCT without fear of stigmatisation from colleagues.

\section{Implications for practice}

The developments in HIV medicine have contributed to an increase in the number of people living with HIV as mortality has decreased (Ford et al. 2012:1470). In addition, efforts have increased over the years to fight the stigma that comes with being diagnosed with HIV. Despite improved lifestyle and less stigmatisation in the general populace, stigmatisation amongst healthcare workers persists. Healthcare workers need the support of institutional management for HIV testing in order to avoid the stigma imposed on them by colleagues and the community. In order to achieve positive outcomes for healthcare workers, the infrastructure of the healthcare institutions needs to be changed in order to provide healthcare workers with privacy for counselling and the space for group support and meetings (El-Sadr \& Abrams 2007:S67).

\section{Limitations of the study}

The study was conducted in two healthcare settings in the Tshwane district of South Africa under the provincial government and excluded local government health settings. Furthermore, the study included only 26 health workers and thus similar studies need to be conducted in other healthcare settings in the Tshwane district of South Africa in order to be able to use the findings for the population of all healthcare workers in Tshwane.

\section{Recommendations}

The following recommendations emerged:

- There is a need to establish accessible HIV workplace programmes for health workers.

- The management needs to support healthcare workers who are living with HIV and AIDS.

- Training of healthcare workers as recipients of HCT and PICT is indicated from the findings.

\section{Conclusions}

Healthcare workers such as nurses and VCT counsellors are involved in the enormous task of facilitating South Africa in its journey toward zero new HIV infections through the implementation of HCT and PICT. People with HIV infections are increasingly being identified and treated and fewer new infections are manifesting (DoH 2012:12). Zero new infections are still a way off, but are considered achievable. However, healthcare workers themselves are not accessing their own health services because of various challenges. They have become the newly infected that need treatment. Even though healthcare workers provide HCT, VCT and PICT, they observe patients being stigmatised and are thus reluctant to use PICT. They, in turn, behave like many of their clients before receiving counselling, notwithstanding the vast knowledge that they possess regarding the benefits of HCT uptake. The need for special dedicated sites where healthcare workers could access HCT privately cannot be overlooked as healthcare workers need to live healthy lives in order to continue providing HCT, VCT and PICT to clients. Healthcare workers need to be healthy and thus be role models in order to motivate clients to undergo HCT and access ARVs. The findings of this study also indicate that a programme is necessary in order to train healthcare workers on how to access HCT for themselves and their peers without fear. A gap exists in current methods: healthcare workers are only viewed as agents and not recipients when, in reality, they too need systems in place to allow them to be recipients of HCT.

\section{Acknowledgements}

The authors acknowledge Ms S.S. Phiri, who had a part in the writing of the proposal, participants from Tshwane and Jubilee District Hospitals, Dr M. Chinuoya for her professional contribution in collecting data and writing the article and the department of Nursing of University of Pretoria for affording the authors an opportunity to undertake the study.

\section{Competing interests}

The authors declare that they have no financial or personal relationship(s) which may have inappropriately influenced them in writing this article.

\section{Authors' contributions}

M.S.M. and M.D.P. (University of Pretoria) wrote the proposal, conducted the data analysis and undertook the 
writing of the article. F.M.M. (University of Pretoria) was involved in the writing of the article and conducted the critical review, R.N.N. (University of Pretoria) and M.C. (Northumbria University) were involved in the collection of data and writing of the article and R.R. (University of Pretoria) was involved with data analysis and the writing of the article.

\section{References}

Akinboro, A.A., Adejumo O.P. \& Olowekere E.A., 2012, 'Community health care workers' risk perception of occupational exposure to HIV in Ibadan, south-west Nigeria', African Journal of Primary Health Care \& Family Medicine; 4(1), 9 pages. http://dx.doi.org/10.4102/phcfm.v4i1.338

Conference Report, Arusha, February 2012, 'Sustaining employers' organisation response to HIV and AIDS in the workplace in Africa: compilation of experiences and best practices', International Organisation of Employers (IOE).

Connelly, D., Veriava, Y., Roberts, S., Tsotetsi, J., Jordan, A., DeSilva, E., et al., 2007 'Prevalence of HIV infection and median CD4 counts among health care workers in South Africa', South African Medical Journal, 97(2), 115-120.

Davhana-Maselesele, M. \& Igumbor, J.O., 2008, 'The impact of caring for persons living with HIV and AIDS on the mental health of nurses in the Limpopo Province',
Curationis, 31(2), 67-73. http://dx.doi.org/10.4102/curationis.v31i2.987

Department of Health [DoH], 2010, HIV counselling and testing (HCT) policy guidelines, Pretoria, Government Printers.

Department of Health [DoH], 2012, National strategic plan on HIV, STIs and TB: 2012 2016, Pretoria, Government Printers.

Duber, H.C., Coates, T.J., Szekeras, G., Kaji, A.H. \& Lewis, R.J. 2010, 'Is there an association between PEPFAR funding and improvement in national health indicators in Africa? A retrospective study', Journal of the International AIDS Society, 13, 9 pages. http://dx.doi.org/10.1186/1758-2652

El-Sadr, W.F. \& Abrams, E.J., 2007, 'Scale up of HIV care and treatment: can it transform healthcare services in resource-limited settings', AIDS, 21(Suppl 5), S65-S70. http://dx.doi.org/10.1097/01.aids.0000298105.79484.62

Ford, N., Singh, K., Cooke, G.S., Mills, E.J., von Schoen-Angerer, T., Kamarulzaman, A. et al., 2012, 'Expanding access to treatment for hepatitis $C$ in resource-limited settings: lessons from HIV/AIDS', Clinical Infectious Diseases, 54(10), 1465-1472. settings: lessons from HIV/AIDS', Clinical
http://dx.doi.org/10.1093/cid/cis227

Gruskin, S., Ahmed, S. \& Ferguson, L., 2008, 'Provider-initiated HIV testing and counselling in health facilities - what does this mean for the health and human rights of pregnant women?' Developing World Bioethics, 8(1), 23-32. http:// dx.doi.org/10.1111/j.1471-8847.2007.00222.x

Hobbs, E., 2011, HIV counselling and testing campaign: The way forward?, viewed 18 October 2013, from http://www.ngopulse.org/article/hiv-counselling-andtesting-campaign-way-forward

Horwood, C., Vermaak, K., Rollins, N., Haskins, L., Nkosi, P. \& Qazi, S., 2009 'Paediatric HIV management at primary care level: an evaluation of the integrated management of childhood illness (IMCI) guidelines for HIV', BMC Pediatrics, 9, 59, 11 pages. http://dx.doi.org/10.1186/1471-2431-9-59

Kalichman, S.C. \& Simbayi, L.C., 2003, 'HIV testing attitudes, AIDS stigma, and voluntary HIV counselling and testing in a black township in Cape Town, South Africa' Sexually Transmitted Infections, 79(6), 442-447. http://dx.doi.org/10.1136/ sti.79.6.442

Kardas-Nelson, M., 2012, How to tackle HIV stigma among health care workers, viewed 18 October 2013, from http://www.aidsmap.com/How-to-tackle-stigmaamong-health-care-workers/page/2454641/

Leon, N.H., Colvin, C.J., Lewin, S., Mathews, C. \& Jennings, K., 2010, 'Provider-initiated testing and counselling for HIV - from debate to implementation', South African Medical Journal, 100(4), 220-221.
Munjanja, O.K., Kibuka, S. \& Dovlo, D., 2005, The nursing workforce in sub-Saharan Africa, Issue Paper 7 , viewed 4 January 2014, from http://www.icn.ch/images/ stories/documents/publications/GNRI/Issue7_SSA.pdf

Makhunga-Ramfolo, N., Chidarikire, T., Farirai, T. \& Matji, R., 2011, 'Provider-initiated counselling and testing (PICT): an overview', Southern African Journal of HIV Medicine, 12(2), 6-11.

Mavedzenge, S.N., Baggaley, R., Ru Lo, Y. \& Corbett, L., 2011, HIV self-testing among health workers: a review of the literature and discussion of current practices, issues and options for increasing access to HIV testing in sub-Saharan Africa, Geneva, World Health Organization.

Musheke, M., Ntalasha, H., Gari, S., McKenzie, O., Bond, V., Martin-Hilber, A., et al., 2013, 'A systematic review of qualitative findings on factors enabling and deterring uptake of HIV testing in sub-Saharan Africa', BMC Public Health, 13, 220, 16 pages. http://dx.doi.org/10.1186/1471-2458-13-220

Nsubunga, F.M \& Jaakkola, M.S., 2005, 'Needle stick injuries among nurses in subSaharan Africa', Tropical Medicine and International Health, 10(8), 773-781.

Obermeyer, C.M. \& Osborn, M., 2007, 'The utilization of testing and counseling for HIV: a review of the social and behavioural evidence', The American Journal of Public Health, 97(10), 1762-1774. http://dx doi.org/10.2105/AJPH 2006.096263

Peu, M.D., van Wyk, N.C. \& Botha, A.D.H., 2008, 'Health promotion needs of Hammanskraal families with adolescents orphaned by HIV/AIDS', Health SA Gesondheid, 13(1), 14-28. http://dx.doi.org/10.4102/hsag.v13i1.254

Pope, D.S., Deluca, A.N., Kali, P., Hausler, H., Sheard, C., Hoosain, E., et al., 2008, 'A cluster-randomized trial of provider-initiated (opt-out) HIV counseling and testing of tuberculosis patients in South Africa', Journal of Acquired Immune Deficiency Syndromes, 48(2), 190-195. http://dx.doi.org/10.1097/QAl.0b013e3181775926

Ranmuthugala, G., Cunningham, F.C., Plumb, J.J., Long, J., Georgiou, A., Westbrook, J.I., et al., 2011, 'A realist evaluation of the role of communities of practice in changing healthcare practice', Implementation Science, $6,49,16$ pages. http:// dx.doi.org/10.1186/1748-5908-6-49

Ritchie, J. \& Spencer, L., 1993, 'Qualitative data analysis for applied policy research' in A. Bryan, \& R. Burges (eds.), Analysing qualitative data, pp. 173-194, London, Routledge.

Rohleder, P. \& Swartz, L., 2005, “"What I've noticed what they need is the stats": lay HIV counsellors' reports of working in a task-orientated health care system', AIDS Care, 17(3), 397-406. http://dx.doi.org/10.1080/09540120512331314376

Smit, R., 2005, 'HIV/AIDS and the workplace: perceptions of nurses in a public hospital in South Africa', Journal of Advanced Nursing, 51(1), 22-29. http://dx.doi. org/10.1111/j.1365- 2648.2005.03456.x

Soai, M., 2012, Distance, time and healthcare workers' attitudes: how they determine people's views towards the healthcare system, viewed 15 April 2013, from http:// www.consultancyafrica.com//index.php?option=com content\&view=article\& $\mathrm{d}=1068$ :distance-time-and-healthcare-workers-attitudes-how-they-determinepeoples-views-towards-the-healthcare-system \&catid=61:hiv-aids-discussionpapers\&Itemid $=268$

Topp, S.M., Li M.S., Chipukuma, J.M., Chiko, M.M., Matongo, E., Bolton-Moore, C., et al., 2012, 'Does provider-initiated counselling and testing (PITC) strengthen early al., 2012, 'Does provider-initiated counselling and testing (PITC) strengthen early HIV-positive patients in Lusaka, Zambia', Journal of the International AIDS Society, HIV-positive patients in Lusaka, Zambia', Journal of the International
15(2), 17352, 9 pages. http://dx.doi.org/10.7448/IAS.15.2.17352

Wenger, E., McDermott, R. \& Snyder, W.M., 2002, Cultivating communities of practice, Boston, Harvard Business Review Press.

Wheelock, A., Eisingerich, A.B., Gomez, G.B., Gray, E., Dybul, M.R. \& Piot, P., 2012, 'Views of policymakers, healthcare workers and NGOs on HIV pre-exposure prophylaxis (PrEP): a multinational qualitative study', BMJ Open, 2(4), e001234, 13 pages. http://dx.doi.org/10.1136/bmjopen-2012-001234

World Health Organization, 2007, Guidance on provider-initiated HIV testing and counselling in health facilities, Geneva, World Health Organization.

World Health Organization, 2011, Progress report 2011: Global HIV/AIDS response. Epidemic update and health sector progress towards universal access, Geneva, World Health Organization.

Zungu, L.I. \& Sanni, B.A., 2011, 'Acceptance and uptake of voluntary HIV testing among healthcare workers in a South African public hospital', South African Family Practice, 53(5), 488-494. 